\title{
Seismology of the lower mantle and core-mantle boundary
}

\author{
Thorne Lay \\ Institute of Tectonics, University of California, Santa Cruz
}

\section{Introduction}

The thermal and chemical processes operating near the core-mantle boundary (CMB) play a major role in the planet's evolution, influencing the magnetic field behavior, chemical cycling in the mantle, irregularities in the rotation and gravitation of the planet, and the configuration of mantle convection. The past decade has witnessed extensive investigation of the core-mantle transition zone using seismological, geodynamical, geomagnetic, and mineral physics - experimental techniques. The many multidisciplinary contributions on this region have been surveyed in several recent review articles (e.g. Loper and Lay, 1994; Jeanloz and Lay, 1993; Bloxham and Jackson, 1991) which provide a comprehensive survey of the recent literature. I confine my attention here to recent seismological evidence for laterally heterogeneous structure in the $D^{\prime \prime}$ region, the lowermost 200 $\mathrm{km}$ of the mantle. While seismic models for $\mathrm{D}^{\prime \prime}$ are still low resolution, we have attained general constraints on the extent of heterogeneity which raise provocative questions as to the origins of the complex structures. The idea that chemical and thermal heterogeneity in $\mathrm{D}$ " is a manifestation of boundary layer processes is a long-standing one (Bullen, 1949), but we are far short of quantifying this notion. In particular, it is not known whether heterogeneities in $D^{\prime \prime}$ are relics of subducted slabs, in-situ accumulations of coremantle chemical reaction products, primordial residuum from the core-formation process, or thermal heterogeneities within a hot, unstable thermal boundary layer.

Travel time studies early in this century established that there are general reductions in the gradients of both $\mathrm{P}$ and $\mathrm{S}$ wave velocities with depth into the $D^{\prime \prime}$ layer. This is apparent in modern average Earth models such as PREM (Dziewonski and Anderson, 1981), as well as in freeoscillation based models (e.g. Kumagai et al., 1992). The reduced seismic-velocity gradients are often associated with a rapid temperature increase within a thermal boundary layer in $D^{\prime \prime}$, the presence of which is required by the existence of core convection. However, Young and Lay (1987) review many seismological studies of the CMB, finding that, as is true near the Earth's surface, one-dimensional models do not suffice to characterize the $D^{\prime \prime}$ region. The inference of strong heterogeneity in the seismic structure has placed an emphasis on imaging three-dimensional variations in $\mathrm{D}^{\prime \prime}$.

During the past decade, three-dimensional aspherical models of lower mantle structure have been constructed using

\section{Copyright 1995 by the American Geophysical Union.}

Paper number 95RG00249.

8755-1209/95/95RG-00249\$15.00 millions of arrival time observations from earthquake location catalogs, as well as sparser, but more precise information from thousands of long-period seismic waveforms, long-period travel times and differential travel times measured from analog and digital recordings, and free oscillation structural coefficients (e.g. Masters et al., 1992; Su and Dziewonski, 1991, 1992; Pulliam et al., 1993; Su et al., 1994). While the resolution of such models is presently only on the scale of $2000-4000 \mathrm{~km}$ laterally and about 200 $\mathrm{km}$ radially, a fundamental revelation is that there is significant large-scale $P$ and $S$ wave seismic velocity heterogeneity within the lowermost mantle. This is a very intriguing result, as these scales are significantly larger than expected for an unstable, hot thermal boundary layer. It appears that either the thermal properties of the $D^{\prime \prime}$ region are strongly influenced by large-scale mantle flow, or there are chemical heterogeneities in the region. There is poor correspondence between large-scale features in $\mathrm{P}$ and $\mathrm{S}$ wave velocities in the current generation of seismic models, which may indicate that thermal variations are not the dominant cause of velocity heterogeneity. However; the current $\mathrm{S}$ velocity models appear more reliable than the $\mathrm{P}$ velocity models, thus this issue awaits the results of ongoing efforts to obtain improved $\mathrm{P}$ velocity models.

The general tendency in global tomographic models is for lower mantle regions below circum-Pacific subduction zones to have higher than average $S$ velocities. There are comparably large-scale low-velocity regions under the central Pacific and beneath Africa, labeled by $\mathrm{Su}$ et al. (1994) the Equatorial Pacific Plume Group and the Great African Plume, respectively, reflecting a general association between $D^{\prime \prime}$ low-velocity structure and concentrations of hotspots at the surface. These gross features tend to be fairly robust between models, while finer features differ significantly, thus there remains substantial uncertainty in the configuration of even the large-scale heterogeneity in D". The shear velocity variations in the tomography models are on the order of $\pm 2.0 \%$, a range compatible with most diffracted wave and differential travel time studies, although localized variations as strong as $\pm 4 \%$ are discussed below. The lateral variations in $\mathrm{D}^{\prime \prime}$ appear to be stronger than those in the overlying mantle in most models, although $\mathrm{Su}$ et al. (1994) argue that below $1700 \mathrm{~km}$ heterogeneity varies little with depth and is dominated by spherical harmonic degrees 2 and 3. In the Su et al. (1994) model there is a tendency for vertical continuity of $S$ velocity heterogeneities upward from the $D^{\prime \prime}$ region into the mid-mantle. This suggests that largescale mantle convection is related to the D" heterogeneity. The general pattern of circum-Pacific fast-velocity material has sometimes been associated with down-wellings related to contemporary subduction (e.g. Richards and Engebretsen, 
1992; Grand, 1994), although some of the D" anomalies are located in regions which have not had substantial subducted slab accumulation (such as the southern Pacific).

While global tomographic inversions continue to improve in resolution and stability, they are still far from revealing CMB processes. Seismological approaches have also been applied to determine finer-scale structures embedded within the large-scale variations, mainly by using detailed body-wave analysis. Waveforms of 10-20 s period body waves have been extensively analyzed to resolve structures with scale lengths of $500-2000 \mathrm{~km}$, while broadband and short-period seismic waves have been studied, primarily using array analyses, to investigate structures from $10-500 \mathrm{~km}$ in scale. A broadband description of the heterogeneity spectrum in $\mathrm{D}^{\prime \prime}$ is emerging, although it is not yet possible to attain global sampling of all scale lengths.

At the intermediate scale lengths sensed by 10-20 s period body waves a wide variety of methods provide information about the CMB region. What has changed most profoundly in the past few years is the quality and size of the data sets that are being investigated, and the ability to account for shallower mantle heterogeneity using the largescale three-dimensional velocity models that are now available. For example, contemporary studies of diffracted $\mathbf{P}$ and $S$ waves (e.g. Wysession et al., 1992) use extensive data sets, modern synthetic waveform-modeling capabilities, and corrections for upper and mid-mantle heterogeneity in the analysis. Diffracted-wave studies generally support the global tomographic inversions in detecting large-scale regions of faster or slower velocity, with $\mathrm{P}$ and $\mathrm{S}$ velocity variations sometimes correlated and sometimes not. Lateral variations as large as $4 \%$ have been found for both $\mathrm{P}$ and $\mathrm{S}$ wave velocities, with inferred $4 \%$ variations in Poisson's ratio.

More localized sampling of D" structure can be obtained by analyzing travel times of core-reflected phases such as $\mathrm{PcP}$ and $\mathrm{ScS}$, usually referenced to direct $\mathrm{P}$ and $\mathrm{S}$ in order to suppress near-source and near-receiver heterogeneity. Phases traversing the relatively uniform outer core, such as SKS and PKP are also used to isolate CMB heterogeneity. Such differential times tend to have relatively good lateral resolution, but poor radial resolution, and large data sets are needed to suppress contributions from shallow mantle heterogeneity. Analyses of various sets of differential times (e.g. Woodward and Masters, 1991; Gaherty and Lay, 1992; Tanaka and Hamaguchi, 1992; Garnero and Helmberger, 1993; Song and Helmberger, 1993; Wysession et al., 1994) have addressed both localized regions of $\mathrm{D}^{\prime \prime}$ as well as global coverage. These travel-time studies are supportive of the presence of significant large-scale heterogeneities on the order of several percent that dominate the overall variations. The differential residuals often display substantially greater variations than predicted by the tomographic models, indicative of strong heterogeneity on scale lengths less than 2000 km (e.g. Garnero and Helmberger, 1993).

While travel-time measurements provide integral constraints on the heterogeneity above the CMB, analyses of seismic waveforms can provide higher resolution of velocity gradients and discontinuous structures. Vidale and Benz (1992) use the simple waveform character of short-period ScP observations to demonstrate that in at least one region the CMB itself is very sharp and lacks any significant transition zone. Broadband observations of S-wave reflections from the core also indicate a simple reflecting boundary in some regions (e.g. Weber, 1993). Amplitudes of PcP and
ScS phases reflected from the CMB tend not to be very diagnostic of average $D^{\prime \prime}$ structure, but the scatter in these phases may bound possible CMB topography (e.g. Neuberg and Wahr, 1991; Rekdal and Doornbos, 1992; Vidale and Benz, 1992; Emmerich, 1993). It remains very difficult to correct for amplitude effects of mantle and receiver heterogeneity, so tight constraints on CMB topography have not yet been achieved using waveforms. Similarly, attempts to constrain CMB topography using travel time data sets have not resulted in any consistent model for the boundary (see Loper and Lay, 1994 for discussion).

Seismic waves that graze the CMB are particularly sensitive to fine structure in $\mathrm{D}^{\prime}$. Wide-angle ScS reflections exhibit waveform complexities that can be interpreted as either the result of high velocity gradients above the CMB or possible anisotropy in the D" layer (Lay and Young, 1991). It appears that most of the lower mantle does not have significant anisotropy, but there is some evidence for anisotropic effects in diffracted $S$ waves, which extensively sample D" (Vinnik et al., 1989). S waves incident on the $\mathrm{CMB}$ at angles such that the reflected $\mathrm{P}$ energy diffracts along the boundary can couple with the transmitted SKS phase to produce SPdiffKS. Analysis of the differential times of SPdiffKS-SKS, which are sensitive to the P-wave velocity at the $C M B$, indicates reductions in $P$ velocity by as much as $5 \%$ over a $100 \mathrm{~km}$ thick layer in $\mathrm{D}^{\prime \prime}$ for some paths (Garnero et al., 1993a). There is an associated rapid change in the SKKS/SKS amplitude ratio near $107^{\circ}$ which has been used to map $3.5 \%$ lateral variations in $\mathrm{P}$ velocity at the CMB (Silver and Bina, 1993). The implications of these studies are very different than those of Vidale and Benz (1992), and the results of any one study should not be generalized to apply to the entire CMB. Overall, grazing waves provide evidence for a wide range of velocity structures just above the $\mathrm{CMB}$, involving both strong velocity reductions and possibly strong velocity increases or anisotropy, which have just begun to be mapped out.

One of the enduring fundamental questions about the D" region is whether it is dynamically separate from the overlying lower mantle, or simply a zone of increased heterogeneity at the base of a large convecting layer. Over the past two decades numerous seismic waveform studies have indicated that a globally extensive, relatively abrupt velocity increase exists several hundred kilometers above the CMB, which is sometimes invoked as evidence for a distinct D" layer. Detailed travel time, ray parameter, and seismic waveform studies have provided evidence for arrivals that reflect from the increased velocity gradient about 150 $300 \mathrm{~km}$ above the core. Recent P-wave studies have mainly used short-period and broadband array data (e.g. Weber and Körnig, 1992; Neuberg and Wahr, 1991; Houard and Nataf, 1992, 1993; Nataf and Houard, 1993; Krüger et al., 1993, 1994; Weber, 1993; Yamada and Nakanishi, 1993; Vidale and Benz, 1993). Recent S-wave studies have made extensive use of long-period WWSSN and broadband digital data (e.g. Young and Lay, 1990; Revenaugh and Jordan, 1991; Gaherty and Lay, 1992; Garnero et al, 1993b; Weber, 1993; Kendall and Shearer, 1994). Synthetic waveform modeling for locally layered media has been extensively used to interpret the extra arrivals as triplications from 1.5$3.0 \%$ velocity increases, usually assumed to be sharp discontinuities although transition zones up to several $10 \mathrm{~s}$ of kilometers thick cannot be ruled out. A 3\% increase is larger than any other structure below the transition zone. 
Velocity discontinuities with less than $1 \%$ velocity increase would not be detectable with most data sets. It appears unlikely that the several percent velocity fluctuations in $\mathrm{D}^{\text {" }}$ are superimposed on a background average model with a 2$3 \%$ fast $D^{\prime \prime}$ layer; rather it is more consistent with global travel time data to visualize there being several percent fluctuations around a PREM-type model, with localized regions of $D^{\prime \prime}$ being fast, accompanied by a concentrated velocity increase.

The regions exhibiting either $\mathrm{P}$ or $\mathrm{S}$ discontinuities in D" are widely distributed (see Nataf and Houard, 1993, for a summary), with the strongest features found below circumPacific regions. Kendall and Shearer (1994) have obtained the most extensive global coverage by searching for precritical shear-wave reflections in long-period digital data, finding evidence for discontinuities beneath Australia, North America, and northern Africa in addition to under Pacific margins. The shear wave reflector is found at variable depths from $150-350 \mathrm{~km}$ above the $\mathrm{CMB}$, with a median depth around $250 \mathrm{~km}$. It is possible that more than one discontinuity structure may be present in some locations, as suggested by Gaherty and Lay (1992), who model some observations with a pair of discontinuities 150 and $300 \mathrm{~km}$ above the CMB rather than a single reflector. Strengthening or weakening of one or the other of the discontinuities could give the appearance of topography on the boundary. Complexity of the triplicated phases may alternatively arise from lateral truncation of a single discontinuity (Weber, 1993).

There are many uncertainties regarding the nature of the extra arrivals and their interpretation in terms of triplications from a high velocity layer in D". There are certainly high quality observations that lack any evidence of extra arrivals, for both $\mathrm{P}$ and $\mathrm{S}$ waves. Thus, $\mathrm{P}$ and $\mathrm{S}$ wavefields can have variable manifestations, usually with $P$-wave energy showing more intermittent and generally weaker reflections from $D^{\prime \prime}$ than $S$ waves. Since the P-wave observations involve higher frequencies it may be easiest to explain this by invoking a velocity gradient at the top of $D$ " rather than a sharp discontinuity, with variations in the strength of the gradient accounting for the frequencydependent effects. Alternatively, a complex surface with topography on it will show high variability in short-period reflections, but greater stability for long-period signals. Either model may account for rapid lateral variations in the strength of energy reflected from the D" layer. This complicates interpretation of observations lacking extra arrivals (e.g. Krüger et al., 1993; Weber, 1993), as there are many ways in which a coherent reflection can be prevented or obscured, or conversely, reflections may be locally augmented, leading to misinterpretations involving either too smooth of a structure or a stronger discontinuity than is actually present. The only current approach to resolving this problem is to increase the data coverage, using all available source-station combinations. Weber (1993) analyzed 255 events at suitable distances recorded by the GRF broadband array, finding 74 events with extra arrivals, 120 with no D" reflection and 61 borderline cases. There are regions as large as $1500 \mathrm{~km}$ (Young and Lay, 1990) and as small as $130 \mathrm{~km}$ (Weber, 1993) over which there appears to be a relatively uniform D" discontinuity structure, but the intermittence of the structure, or at least of coherent reflections from it, is perplexing. One cannot preclude the possibility that the seismic waves are merely detecting large-scale modula- tions of ubiquitous small-scale heterogeneity in the region, with seemingly coherent reflections being produced by constructive interference. Comparable uncertainty exists with respect to reflections from the deep crust, and the available data for studying structures $2800 \mathrm{~km}$ deeper are far more limited. The issue of whether $D^{\prime \prime}$ is a globally extensive distinct layer must be considered to be still open, until more extensive mapping of fine-scale structure in D" is attained.

\section{Discussion}

There appear to be substantial large-scale heterogeneities in the $\mathrm{D}^{\prime \prime}$ region, with volumetric velocity fluctuations of $\pm 2 \%$ or larger. Our knowledge of velocity dependence on temperature at such very high pressures is actually very poor, but extrapolation of experimental constraints from lower pressures suggests that excessively large variations, on the order of $1000 \mathrm{~K}$, would be required for a purely thermal explanation (e.g. Yuen et al., 1993). Coupled with the poor correlation between $\mathrm{P}$ and $\mathrm{S}$ velocity perturbations in many regions, it has become commonplace to assert that there must be a chemical contribution to the observed velocity heterogeneity. At present, this must be deemed a soft conclusion, but even if we assume that it is accurate it is unclear what the nature of the chemical heterogeneity must be.

The geometry of fast velocity regions beneath the circum-Pacific and the tendency for these regions to show the clearest evidence for $D^{\prime \prime}$ velocity discontinuities points toward a causal relationship with mantle convection. The notion that interaction with transition zone phase boundaries tends to cause episodic flushing events of upper mantle slab accumulations (e.g. Tackley et al., 1993) offers one means for accumulating large-scale down-wellings of chemically differentiated slab materials which may sink rather rapidly to the $\mathrm{CMB}$, retaining sufficient thermal anomaly to have faster than average seismic velocities in the 'footprint' of descending material. Chemically distinct components in the slabs, particularly the eclogitic crustal components may provide strong chemical contrasts that could contribute to small-scale heterogeneities in $\mathrm{D}$ ", presumably in combination with small-scale thermal irregularities in the deep boundary layer. Gradual thermal equilibration of the slab material could weaken the velocity anomalies, leaving only weak, intermittent chemical contrasts. Other scenarios such as in situ growth of chemical heterogeneities via coremantle chemical reactions can also be invoked, but suffer from lack of any constraints on the volumes of material that can be produced or on why there may be any relationship to the configuration of the present day shallow mantle convection. A concerted effort to map out the broadband heterogeneity spectrum of the $\mathrm{D}^{\prime}$ region will eventually provide the information for assessing the configuration of thermal and chemical heterogeneity in this region, but the effort will be very challenging. Seismologists will need to develop new processing methods, exploiting the full complexity of secondary arrivals in broadband seismograms and using stacking methods to enhance the signal-to-noise ratio for weak arrivals. New three-dimensional modeling capabilities are also needed to enable modeling of the complex signals from $\mathrm{D}^{\prime}$. In addition, seismological results must be interpreted in the context of independent constraints on the $D^{\prime \prime}$ structure provided by other disciplines, if we are to resolve the processes occurring near the CMB. 
Acknowledgments. T. Lay's research on the core-mantle boundary is currently supported by the National Science Foundation under Grant EAR9305894. Ed Garnero and two anonymous reviewers gave useful editorial comments. This is contribution number 248 of the W. M. Keck Seismological Laboratory of the Institute of Tectonics, University of California at Santa Cruz.

\section{References}

Bloxham, J, and A. Jackson, Fluid flow near the surface of Earth's outer core, Rev. of Geophys., 29, 97-120, 1991.

Bullen, K. E., Compressibility-pressure hypothesis and the Earth's interior, Mon. Not. R. Astr. Soc., Geophys. Suppl., 5, 355-368, 1949.

Dziewonski, A. M., and D. L. Anderson, Preliminary reference Earth model, Phys. Earth Planet. Inter., 25, 297-356, 1981.

Emmerich, H., Theoretical study on the influence of CMB topography on the core reflection, ScS, Phys. Earth Planet. Int., 80, 125-134, 1993.

Gaherty, J. B., and T. Lay, Investigation of laterally heterogeneous shear velocity structure in D" beneath Eurasia, J. Geophys. Res., 97, 417-435, 1992.

Garnero, E. J., and D. V. Helmberger, Travel times of S and SKS: Implications for three-dimensional lower mantle structure beneath the central Pacific, J. Geophys. Res., 98, 8225-8241, 1993.

Garnero, E. J, S. P. Grand, and D. V. Helmberger, Low P-wave velocity at the base of the mantle, Geophys. Res. Lett., 20, 1843-1846, 1993a.

Garnero, E. J., D. V. Helmberger, and S. Grand, Preliminary evidence for a lower mantle shear wave velocity discontinuity beneath the central Pacific, Phys. Earth Planet. Inter., 79, 335-347, 1993b.

Grand, S. P., Mantle shear structure beneath the Americas and surrounding oceans, J. Geophys. Res., 99, 11591-11622, 1994.

Houard, S., and H.-C. Nataf, Further evidence for the 'Lay discontinuity' beneath Northern Siberia and the North Atlantic from short-period Pwaves recorded in France, Phys. Earth Planet. Inter., 72, 264-275, 1992.

Houard, S., and H.-C. Nataf, Laterally varying reflector at the top of D" beneath northern Siberia, Geophys. J. Int., 115, 168-182, 1993.

Jeanloz, R., and T. Lay, The core-mantle boundary, Sci. Am., May, 4855, 1993.

Kendall, J.-M., and P. M. Shearer, Lateral variations in D" thickness from long-period shear-wave data, J. Geophys. Res., 99, 1157511590, 1994.

Krüger, F., M. Weber, F. Scherbaum, and J. Schlittenhardt, Double beam analysis of anomalies in the core-mantle boundary region, Geophys. Res. Lett., 20, 1475-1478, 1993.

Krüger, F., M. Weber, F. Scherbaum, and J. Schlittenhardt, Evidence for normal and inhomogeneous lowermost mantle and core-mantle boundary structure under the Arctic and northern Canada, Geophys. J. Int., in press, 1994.

Kumagai, H., Y. Fukao, N. Suda, and N. Kobayashi, Structure of the D" layer inferred from the Earth's free oscillations, Phys. Earth Planet. Inter., 73, 38-52, 1992.

Lay, T. and D. V. Helmberger, A lower mantle S-wave triplication and the shear velocity structure of D", Geophys. J. R. Astron. Soc., 75, 799-838, 1983.

Lay, T., and C. J. Young, Analysis of seismic SV waves in the core's penumbra, Geophys. Res. Lett., 18, 1373-1376, 1991.

Loper, D. E., and T. Lay, The core-mantle boundary region, $J$. Geophys. Res., in press, 1994

Masters, G., H. Bolton, and P. Shearer, Large-scale 3-dimensional structure of the mantle (abstract), Eos, Trans. AGU, 73, 201, 1992.

Nataf, H.-C., and S. Houard, Seismic discontinuity at the top of D": a world-wide feature?, Geophys. Res. Lett., 20, 2371-2374, 1993.

Neuberg, J., and J. Wahr, Detailed investigation of a spot on the coremantle boundary using digital PcP data, Phys. Earth Planet. Inter., 68, 132-143, 1991

Pulliam, R. J., D. W. Vasco, and L. R. Johnson, Tomographic inversions for mantle $P$ wave velocity structure based on the minimization of $1^{2}$ and $1^{1}$ norms of international seismological centre travel time residuals, J. Geophys. Res., 98, 699-734, 1993.

Rekdal, T., and D. J. Doornbos, The times and amplitudes of core phases for a variable core-mantle boundary layer, Geophys. J. Int. 108, 546-556, 1992.

Revenaugh, J. and T. H. Jordan, Mantle layering from ScS reverberations. 4. The lower mantle and core-mantle boundary, $J$. Geophys. Res., 96, 19811-19824, 1991.

Richards, M. A., and D. C. Engebretsen, Large-scale mantle convection and the history of subduction, Science, 355, 437-440, 1992.

Silver, P. G., and C. Bina, An anomaly in the amplitude ratio of SKKS/SKS in the range $100-108^{\circ}$ from portable teleseismic data, Geophys. Res. Lett., 20, 1135-1138, 1993.

Song, X., and D. V. Helmberger, Effect of velocity structure in D" on PKP phases, Geophys. Res. Lett., 20, 285-288, 1993.

Su, W.-J., and Dziewonski, A. M., Predominance of long-wavelength heterogeneity in the mantle, Nature, 352, 121-126, 1991.

Su, W.-J., and Dziewonski, A. M., On the scale of mantle heterogeneity, Phys. Earth and Planet. Inter., 74, 29-54, 1992.

Su, W.-J., Woodward, R. L., and Dziewonski, A. M., Degree-12 model of shear velocity heterogeneity in the mantle, J. Geophys. Res., 99, 6945-6980, 1994.

Tackley, P. J., D. J. Stevenson, G. Glatzmaier, and G. Schubert, Effects of an endothermic phase transition at $670 \mathrm{~km}$ depth on spherical mantle convection, Nature, 361, 699-704, 1993.

Tanaka, S., and H. Hamaguchi, Heterogeneity in the lower mantle beneath Africa, as revealed from S and ScS phases, Tectonophys., 209, 213-222, 1992.

Vidale, J. E., and H. M. Benz, A sharp and simple section of the coremantle boundary, Nature, 359, 627-629, 1992.

Vidale, J. E., and H. M. Benz, Seismological mapping of fine structure near the base of the Earth's mantle, Nature, 361, 529-532, 1993

Vinnik, L. P., V. Farra, and B. Romanowicz, Observational evidence for diffracted SV in the shadow of the Earth's core, Geophys. Res. Lett., 16, 519-522, 1989.

Weber, M., P- and S-wave reflections from anomalies in the lowermost mantle, Geophys. J. Int., 115, 183-210, 1993.

Weber, M., and M. Körnig, A search for anomalies in the lowermost mantle using seismic bulletins, Phys. Earth Planet. Inter., 73, 1-28. 1992.

Woodward, R. L., and G. Masters, Lower mantle structure from ScS-S differential travel times, Nature, 352, 231-233, 1991.

Wysession, M. E., E. A. Okal, and C. R. Bina, The structure of the core mantle boundary from diffracted waves, J. Geophys. Res., 97, 87498764, 1992.

Wysession, M. E., L. Bartko, and J. Wilson, Investigating the coremantle boundary using GDSN core-reflected shear waves, $J$. Geophys. Res., 99, 13667-13684, 1994.

Yamada, A., and I. Nakanishi, The density jump across the ICB and constraints on P-reflector in the D" layer from observation of the 1992 Chinese nuclear explosion, Geophys. Res. Lett., 20, 2195-2198, 1993.

Young, C. J., and T. Lay, The core-mantle boundary, Ann. Rev. Earth Planet. Sci., 15, 25-46, 1987.

Young, C. J., and T. Lay, Multiple phase analysis of the shear velocity structure in the D" region beneath Alaska, J. Geophys. Res., 95, 17385-17402, 1990.

Yuen, D. A., O. Cadek, A. Chopelas, and C. Matyska, Geophysical inferences of thermal-chemical structures in the lower mantle, Geophys. Res. Lett., 20, 899-902, 1993.

T. Lay, Earth Sciences Department, Earth and Marine Science Building, University of California, Santa Cruz, CA 95064 (e-mail: thorne@earthsci.ucsc.edu)

(Received June 10, 1994; accepted November 21, 1994.) 